\title{
Hybrid Bistability in Nano-opto-mechanical Metamaterial
}

\author{
Dimitrios Papas', Jun-Yu Ou' ${ }^{1}$, Eric Plum¹, and Nikolay I. Zheludev ${ }^{1,2}$ \\ 1. Optoelectronics Research Centre and Centre for Photonic Metamaterials, University of Southampton, Southampton, SO17 1BJ, UK \\ 2. Centre for Disruptive Photonic Technologies, SPMS, TPI, Nanyang Technological University, Singapore, 637371, Singapore \\ Authore-mail address:D.Papas@soton.ac.uk,erp@orc.soton.ac.uk,niz@orc.soton.ac.uk
}

\begin{abstract}
A nanowire array decorated with plasmonic resonators acts as optically bistable device. The optical properties of this metamaterial exhibit hysteresis and bistability when it is driven by a piezo actuator across its mechanical resonance frequency. (C) 2020 The Author(s)
\end{abstract}

\section{Introduction}

In mechanical systems nonlinearity is much easier to achieve than in optical systems, e.g. a gravitational pendulum or a string are highly nonlinear devices. This can be used to attain a memory function, where the memory states are based on bistability of optical properties of a nano-opto-mechanical metamaterial while driving its mechanical subsystem.

Here we show that vibrations of an array of nanowires decorated with plasmonic resonators exhibit hysteresis resulting in bistability of the metamaterial's optical properties when the structure is driven by an ultrasonic piezo actuator across its mechanical resonance (Fig. 1). Reflectivity contrast between the bistable states exceeds one order of magnitude and requires only sub-microwatt ultrasonic driving power levels.

\section{Results}

The bistable structure is a free-standing nanomechanical metamaterial supported by a silicon frame that is attached to a piezoelectric transducer. Application of AC voltage to the piezo element generates ultrasound vibrations that result in modulation of the optical properties of the nanomechanical metamaterial, and such modulation is enhanced at resonance frequencies. When such mechanical resonances are driven in the nonlinear regime, the response of the structure can become dependent on its history of previous excitation, resulting in a hysteresis loop for increasing and decreasing vibration frequencies that originates from different oscillation amplitudes of the nanomechanical structure at the same mechanical frequency $\Omega$ (Fig. 1). We detect the two mechanical states optically, through the optical properties of the resonant plasmonic metamaterial structure. Transmission and reflection of the metamaterial are studied in vacuum $\left(4 \times 10^{-3} \mathrm{mbar}\right)$ with a focused $70 \mu \mathrm{W}$ CW laser beam of frequency $\omega=230 \mathrm{THz}(1310 \mathrm{~nm}$ wavelength) that is polarized parallel to the nanowires (Fig. 2a).

(a)

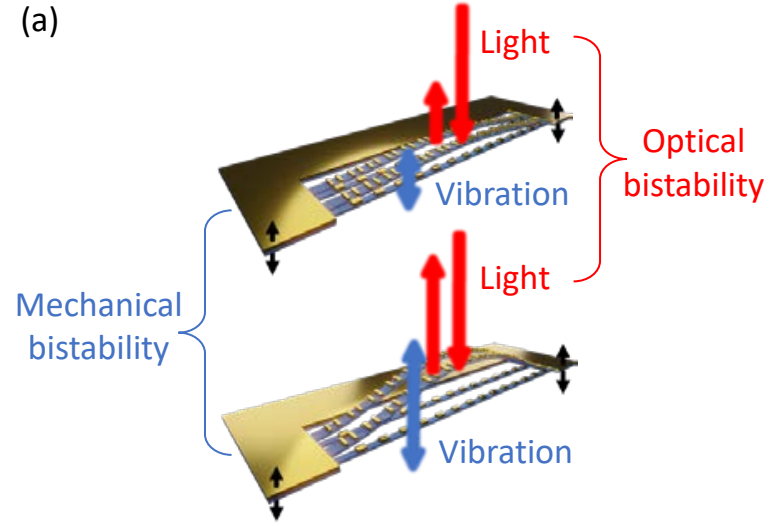

(b)

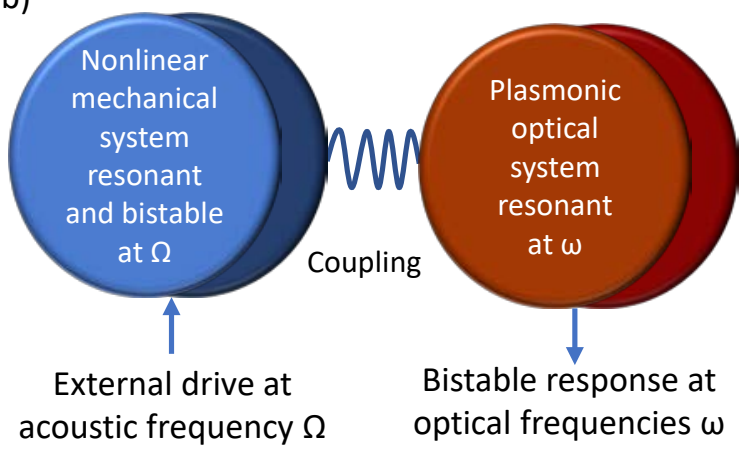

Fig. 1. Optomechanical bistability. (a) Bistable mechanical states of a metamaterial nanostructure cause bistability of its optical properties. (b) Mechanically resonant vibration of the structure yields two stable amplitudes of oscillation for the same ultrasound acoustic driving signal at frequency $\Omega$ in the $\mathrm{MHz}$ range. The mechanical oscillation controls the coupling between the plasmonic resonators that make up the metamaterial and therefore the mechanical bistability is translated into bistable optical properties at frequencies $\omega$ of $100 \mathrm{~s} \mathrm{of} \mathrm{THz.}$

The nanomechanical metamaterial was fabricated on a $100 \mu \mathrm{m}$ x $100 \mu \mathrm{m}$ membrane consisting of 50-nm-thick silicon nitride covered by a 50-nm-thick layer of gold. The gold-coated membrane was structured by focused ion 
beam milling in order to create a metamaterial array of 30 silicon nitride nanowires of $15.4 \mu \mathrm{m}$ length and alternating width, which support П-shaped plasmonic metamolecules with a period of $800 \mathrm{~nm}$ (Fig. 2a). The metamaterial has a resonance at about $\omega=230 \mathrm{THz}$ (1300 nm wavelength) and electromagnetic coupling between the gold bars that make up the П-shaped resonators makes its optical properties sensitive to mutual nanowire displacement.

Fig. 2b shows the modulation depth of the metamaterial's reflectivity when mechanically driven by a sinusoidal signal of frequency $\Omega$, for driving amplitudes from $0.1 \mathrm{~V}_{\mathrm{p} \text {-p }}$ up to $0.6 \mathrm{~V}_{\mathrm{p}-\mathrm{p}}$. With increasing driving voltage, the resonance shifts to higher frequencies, while it's asymmetry and amplitude increase. Most importantly, the reflectivity modulation becomes dependent on whether the excitation frequency $\Omega$ is swept up (black) or down (red). Already at $0.1 \mathrm{~V}_{\mathrm{p}-\mathrm{p}}$, the formation of a bistability loop is observed, which becomes larger at higher voltages. At 0.6 $\mathrm{V}_{\mathrm{p}-\mathrm{p}}$ a maximum optical modulation depth of $22 \%$ is recorded at around $1.624 \mathrm{MHz}$ as the vibration frequency is increased, while modulation of only $1.8 \%$ is observed at the same frequency as the vibration frequency is decreased, corresponding to $12 \mathrm{x}$ optical contrast. In this case, the power dissipated by the piezoelectric transducer is about 100 $\mathrm{mW}$, however, considering that the membrane-metamaterial system is about $3.5 \times 10^{7}$ times lighter than the transducer and supporting frame, we estimate that sub-microwatt mechanical power drives the bistable response of the membrane-metamaterial system. Indeed, the bistable loop is clearly visible at $0.1 \mathrm{~V}_{\mathrm{p}-\mathrm{p}}$ driving voltage, suggesting that $1 \mathrm{nW}$ mechanical power delivered to the membrane may be sufficient to drive the bistable response.

(a)

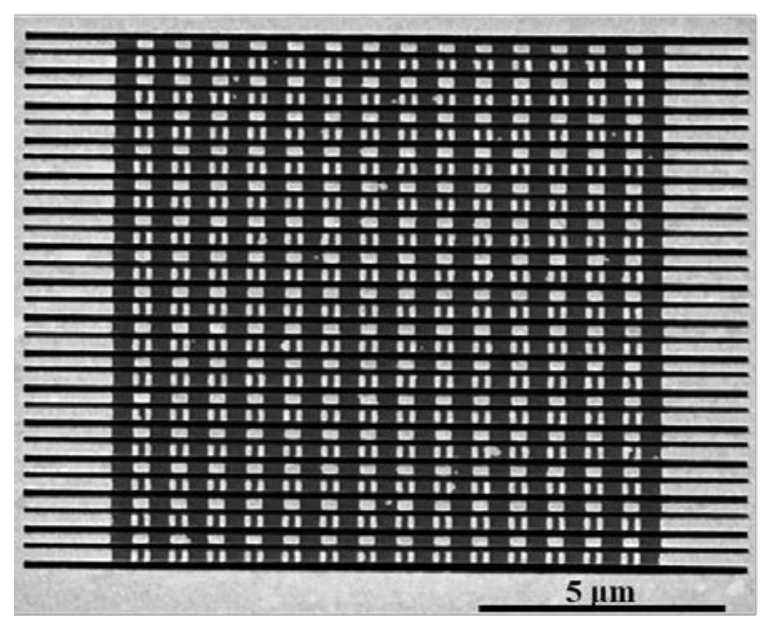

(b)

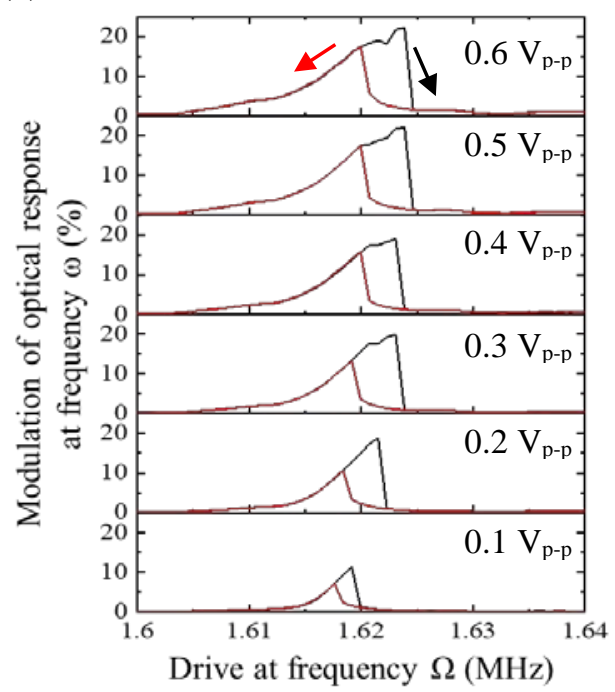

Fig. 2. Bistable metamaterial and its optical response at optical frequency $\omega=230 \mathrm{THz}$ when excited mechanically at frequency $\Omega$. (a) SEM image showing the bistable membrane-based nanomechanical metamaterial. (b) Evolution of the optically detected mechanical resonance located between $\Omega=1.6 \mathrm{MHz}$ and $1.64 \mathrm{MHz}$ for different driving voltage applied to the piezoelectric element. The resonant optical properties measured with increasing (black) and decreasing (red) driving frequency $\Omega$ are different. The structure is bistable, exhibiting different stable optical responses at the same frequency - memory states - that are selected based on the structure's history of excitation.

\section{Summary}

We demonstrate bistable optical properties due to mechanical bistability of a nano-opto-mechanical system. The optical properties of the nanostructure - a nanomechanical metamaterial on a membrane - depend on its history of vibration. We observe $12 x$ reflectivity modulation contrast between the bistable states when driving the system at sub-microwatt power levels and our results suggest that a nanowatt may be sufficient to engage the bistability. This work paves the way towards mechanically nonlinear, optically resonant structures that could serve as low-power binary memory elements in photonic systems. 